\title{
Sortir du jeu. Sur un paradoxe du théâtre de Diderot
}

\section{Marc Escola}

\section{(2) OpenEdition}

1 Journals

Édition électronique

URL : https://journals.openedition.org/edl/3171

DOI : $10.4000 /$ edl. 3171

ISSN : 2296-5084

Éditeur

Université de Lausanne

\section{Édition imprimée}

Date de publication : 24 septembre 2020

Pagination : 97-120

ISBN : 978-2-940331-74-1

ISSN : 0014-2026

\section{Référence électronique}

Marc Escola, "Sortir du jeu. Sur un paradoxe du théâtre de Diderot », Études de lettres [En ligne], 313 | 2020, mis en ligne le 24 septembre 2021, consulté le 25 septembre 2021. URL : http:// journals.openedition.org/edl/3171; DOI : https://doi.org/10.4000/edl.3171 


\section{SORTIR DU JEU. SUR UN PARADOXE DU THÉÂTRE DE DIDEROT}

La réduction de la «scène" au "salon" constitue le leitmotiv des Entretiens qui encadrent Le Fils naturel, premier drame de Diderot (1757). Comment comprendre cette insistance à dénier au théâtre son lieu propre? L'affirmation n'a pas d'autre fin que de permettre la théorisation d'une "déposition » du jeu théâtral, soit «l'exposition» du comédien à une dynamique interne à l'action représentée, qui fait émerger le jeu à l'intérieur des relations entre les personnages où il ne peut être que surpris par celui qui regarde. Ce jeu sans adresse ni direction, c'est celui dont le cinéma a besoin. Tel est le legs de Diderot, identifié par Eisenstein dans un article célèbre. Le jeu serait ainsi le seul domaine où le cinéma doit impérativement s'affranchir de sa filiation avec l'art dramatique, pour se revendiquer comme «fils naturel du théâtre».

De tous les paradoxes que le théâtre de Diderot et ses réflexions sur la théorie dramatique nous offrent à méditer, le plus troublant n'est pas celui auquel l'auteur du Paradoxe sur le comédien a attaché son nom. La thèse de cet essai tardif (1769) fait couler l'encre aussi facilement qu'un acteur sublime laisse échapper des larmes, et peut-être pour les mêmes raisons - pas plus que le comédien dans l'exercice de son jeu, le philosophe n'adhère au propos qui veut que:

C'est l'extrême sensibilité qui fait les acteurs médiocres; c'est la sensibilité médiocre qui fait la multitude des mauvais acteurs; et c'est le manque absolu de sensibilité qui prépare les acteurs sublimes ${ }^{1}$.

I. D. Diderot, Paradoxe sur le comédien, p. 281. Toutes nos citations renvoient à l'édition J. Goldzink en deux volumes (Gf-Flammarion, 2005), qui réunit d'un côté les drames: Le Fils naturel, Le Père de famille, Est-il bon? Est-il méchant?; de l'autre, les essais: Entretiens sur Le Fils naturel, De la poésie dramatique, Paradoxe sur le comédien. 
Les arguments du philosophe, à l'appui de ce que Diderot désigne luimême comme un "beau paradoxe " 2 , pèsent ce que valent les larmes du vrai comédien, lequel:

[...] pleure comme un prêtre incrédule qui prêche la Passion; comme un séducteur aux genoux d'une femme qu'il n'aime pas, mais qu'il veut tromper; [...] ou comme une courtisane qui ne sent rien, mais se pâme entre vos bras ${ }^{3}$.

Le paradoxe ne tient pas davantage dans le fait que l'ensemble formé par le premier des «drames» de Diderot, Le Fils naturel (1757), et par la discussion de ses mérites sous le sceau d'une fiction dialoguée dans les trois Entretiens sur Le Fils naturel, puisse être solidairement regardé comme une "espèce de roman», inassimilable par la scène théâtrale:

Mon dessein n'étant pas de donner [le Fils naturel] au théâtre, je joignis [aux cinq actes de la pièce] quelques idées que j'avais sur la poétique, la musique, la déclamation et la pantomime; et je formai du tout une espèce de roman que j'intitulai «Le Fils naturel, ou les épreuves de la vertu, avec l'histoire véritable de la pièce " 4 .

On prendra d'abord le temps de décrire le dispositif qui favorise, sinon conditionne l'élaboration de ce que Diderot nomme un peu plus tard, avec plus d'audace et sans trop d'hésitations, son «système d'action théâtrale»:

Ma première et ma seconde pièce [Le Fils naturel, 1757 et Le Père de famille, 1758] forment un système d'action théâtrale dont il ne s'agit pas de chicaner un endroit, mais qu'il faut adopter ou rejeter en entier ${ }^{5}$.

2. Lettre à Grimm [commanditaire d'une première version de l'essai], 14 nov. 1769, in D. Diderot, Entretiens sur Le Fils naturel, p. 46.

3. D. Diderot, Paradoxe sur le comédien, p. 281 sq.

4. D. Diderot, De la Poésie dramatique, \$X, p. 200.

5. Lettre à Mme Riccoboni, 27 nov. 1758, in. D. Diderot, Entretiens sur Le Fils naturel, p. 354. 


\section{1. "Et la pièce ne finit pas»}

Les cinq actes du Fils naturel se trouvent encadrés par un prologue et un épilogue qui rapportent les circonstances de la rédaction, puis de la première représentation privée de la pièce, en précisant ultimement le cadre des Entretiens qui porteront, les jours suivants, tout à la fois sur la pièce, sa représentation et sa poétique.

Dans le prologue, un philosophe, que tout invite à assimiler à Diderot lui-même - à commencer par la mention de la parution du sixième volume de l'Encyclopédie, qui permet de dater l'épisode d'octobre 1756 narre à la première personne sa rencontre à la campagne avec un personnage «sombre et mélancolique» nommé Dorval, devenu depuis peu «l'étonnement et l'entretien du canton» à la suite d'un événement dont la nature n'est pas d'emblée précisée. Après avoir entendu, de la bouche même de Dorval, le détail d'une histoire dont le lecteur n'a pas même le sommaire, le philosophe fait valoir que ses épreuves mériteraient de faire l'objet d'un "ouvrage dramatique». Dorval l'informe alors que cette pièce est déjà écrite, qu'il l'a rédigée à la demande de son père Lysimond qui, se sentant près de mourir, a souhaité que l'épisode décisif de l'histoire familiale puisse être consigné dans un texte dramatique pour être rejoué, en manière de commémoration privée, par ses propres protagonistes:

Si tu voulais, ce serait la morale d'une pièce dont une partie de notre vie ferait le sujet, et que nous représenterions entre nous. [...] Il ne s'agit point d'élever ici des tréteaux, mais de conserver la mémoire d'un événement qui nous touche, et de le rendre comme il s'est passé... Nous le renouvellerions nous-mêmes tous les ans, dans cette maison, dans ce salon. Les choses que nous avons dites, nous les redirions. Tes enfants en feraient autant, et les leurs, et leurs descendants. Et je me survivrais à moi-même; et j'irais converser ainsi, d'âge en âge avec tous mes neveux. Dorval, penses-tu qu'un ouvrage qui leur transmettrait nos propres idées, nos vrais sentiments, les discours que nous avons tenus dans une des circonstances les plus importantes de notre vie, ne valût pas mieux que des portraits de famille, qui ne montrent de nous qu'un moment de notre visage? ${ }^{6}$

6. D. Diderot, Le Fils naturel, p. 43. 
Dorval a répondu trop tard au vœu de son père, mort avant d'avoir pu jouer son propre rôle dans la cérémonie commémorative projetée. Le deuil a rejeté un temps la pièce dans l'oubli, mais Dorval s'est laissé convaincre par les autres protagonistes du drame d'en donner une représentation dans le cadre familial, le dimanche suivant. Le philosophe lui extorque une invitation.

Dimanche prochain, nous nous acquittons, pour la première fois, d'une chose qu'ils s'accordent tous à regarder comme un devoir. Ah! Dorval, lui dis-je, si j'osais!

Je vous entends, me répondit-il; mais croyez-vous que ce soit une proposition à faire à Constance, à Clairville, et à Rosalie? Le sujet de la pièce vous est connu; et vous n'aurez pas de peine à croire qu'il y a quelques scènes où la présence d'un étranger gênerait beaucoup. Cependant c'est moi qui fais ranger le salon. Je ne vous promets point, je ne vous refuse pas. Je verrai.

Nous nous séparâmes Dorval et moi: c'était le lundi. Il ne me fit rien dire de toute la semaine. Mais le dimanche matin, il m'écrivit... Aujourd'hui à trois heures précises, à la porte du jardin.

Je m'y rendis. J'entrai dans le salon par la fenêtre; et Dorval, qui avait écarté tout le monde, me plaça dans un coin, d'où, sans être vu, je vis et j'entendis ce qu'on va lire $[\ldots]^{7}$.

Notre lecture des cinq actes de la pièce est donc supposée correspondre à la représentation dont le philosophe a été, par effraction, l'unique spectateur - à une scène près qui est la dernière. La chose nous est signalée à la fin du prologue, qui en diffère l'explication.

[...] je vis et j'entendis ce qu'on va lire, excepté la dernière scène. Une autre fois je dirai pourquoi je n'entendis pas la dernière scène ${ }^{8}$.

L'épilogue nous apprendra pourquoi la représentation n'a pas pu aller à son terme, et s'est brutalement interrompue au seuil de la scène de reconnaissance finale:

J'ai promis de dire pourquoi je n'entendis pas la dernière scène; et le voici. Lysimond n'était plus. On avait engagé un de ses amis, qui

7. Ibid., p. 43 sq.

8. Ibid., p. 44 sq. Viennent à la suite «les Noms des Personnages réels de la Pièce, avec ceux des Acteurs qui pourraient les remplacer", soit la table des dramatis persone, avec le nom des acteurs de la Comédie française souhaités par Diderot. 
était à peu près de son âge, et qui avait sa taille, sa voix et ses cheveux blancs, à le remplacer dans la pièce. Ce vieillard entra dans le salon, comme Lysimond y était entré la première fois, tenu sous les bras par Clairville et par André, et couvert des habits que son ami avait apportés des prisons [anglaises]. Mais à peine y parut-il que, ce moment de l'action remettant sous les yeux de toute la famille un homme qu'elle venait de perdre, et qui lui avait été si respectable et si cher, personne ne put retenir ses larmes. Dorval pleurait, Constance et Clairville pleuraient. Rosalie étouffait ses sanglots et détournait ses regards. Le vieillard qui représentait Lysimond se troubla, et se mit à pleurer aussi. La douleur, passant des maitres aux domestiques, devint générale, et la pièce ne finit pas?

Le paradoxe le plus irritant du théâtre de Diderot tient dans cette interruption de la représentation que Dorval et ses proches se donnent au sein de la fiction du Fils naturel.

Comment comprendre que l'arrivée d'un comédien, fût-il amateur, interrompe le jeu? Entrant pour jouer le rôle qu'on lui a demandé de tenir, il suscite par sa seule apparition une émotion telle, qu'elle contraint tous les autres protagonistes à interrompre la représentation et à sortir du jeu. On peut certes gloser longuement les raisons alléguées par le texte: en se donnant comme le représentant du père, en cherchant à pallier une disparition, le comédien manifeste une absence de façon d'autant plus spectaculaire qu'il a l'apparence du père, et qu'il a revêtu ses habits; par sa seule présence, il interdit d'entretenir ce que Proust ou Deleuze nommeraient "le souvenir pur", en rendant manifeste que le passé n'est plus, qu'il a cessé d'être et qu'il ne saurait plus revenir à l'identique, mais seulement se répéter sur un mode spectral ${ }^{10}$.

\section{Venir sur le plateau}

Je risquerai aujourd'hui une hypothèse d'un autre ordre: si la représentation s'interrompt avec l'entrée d'un comédien, c'est qu'il ne s'agissait pas d'une représentation théâtrale; si le drame familial cesse de se (re) jouer précisément au moment où un comédien entre dans le champ, où

9. Ibid., p. 104.

ı. Cf. M. Escola, «Dans le cristal du temps». 
un homme vient jouer un personnage parmi ceux qui ne jouaient pas, sinon leur propre rôle, en instituant du même coup une scène de théâtre dans l'espace domestique dévolu à la cérémonie privée, c'est que les personnages ne s'adonnaient pas à un petit théâtre de société: ils faisaient du cinéma. Croyant entrer sur une scène, le vieillard s'est avancé sur un plateau de cinéma: il a fallu couper.

L'hypothèse emporte cette autre thèse: tout l'effort théorique de Diderot pour promouvoir un genre et un théâtre, c'est-à-dire un jeu, également nouveaux, est un effort vers le cinéma. Le philosophe a cru inventer un genre - le drame sérieux, genre "moyen" ultérieurement nommé "drame bourgeois", porté sur les fonts baptismaux avec Le Fils naturel, débattu sous couvert de fiction narrative dans les Entretiens sur le Fils naturel et finalement théorisé dans un essai de poétique intitulé De la Poésie dramatique; il se peut toutefois qu'en cherchant un genre, et aiguillonné par Rousseau, contempteur comme lui des spectacles de leur temps ${ }^{11}$, le théoricien ait rencontré un art d'abord impraticable - ce septième art qui devait plus tard s'appeler le cinéma.

L'hypothèse offre au demeurant la seule issue logique à ce qui ne peut manquer d'apparaître comme une inconséquence dans le vœu formulé par le père, qu'il faut ici entendre à nouveau: comment, se sachant à la veille de sa mort, peut-il envisager de «renouveler tous les ans dans cette maison" la cérémonie destinée à "conserver la mémoire [de l']événement»? Sur quel mode envisage-t-il sa propre "survie» pour espérer " [aller] converser d'âge en âge avec tous [s]es neveux"? Comment penser cette répétition ritualisée qui exclut la délégation d'un rôle à un comédien - et en regard de laquelle la pourtant prévisible disparition successive des différents protagonistes semble devoir être tenue pour nulle? De quelle nature peut donc être "l'ouvrage» susceptible de "transmettre» sans variations ni altérations les "idées, sentiments et discours tenus" dans une "circonstance» unique et pourtant réitérable à volonté, de génération en génération et pour les siècles des siècles? La seule façon d'entendre ce vœu, c'est de poser que le père invite à substituer à la galerie de "portraits de famille qui ne montrent qu'un moment de notre

II. Rappelons que les trois ouvrages de Diderot furent rédigés en parallèle dans l'espace de quelques mois seulement, entre 1756 et 1758, pendant que Rousseau préparait, puis publiait de son côté la Lettre à d'Alembert sur les spectacles (1757) (cf. M. Escola, " "Ce théâtre n'est pas le nôtre" "). 
visage", un montage de plusieurs séquences d'un film familial; le jeu n'est pas destiné à être vu (la représentation privée ne prévoit pas de spectateur, et elle ne s'adresse à personne) mais à être filmé.

Ainsi s'expliquerait aussi une autre des anomalies qui émaillent les Entretiens, et qui a beaucoup occupé la critique ${ }^{12}$ : dans son dialogue avec le philosophe spectateur, puis lecteur de la pièce, Dorval se montre toujours soucieux d'une forme de spectacle, alors même qu'il oppose sans cesse le «salon" à la «scène», en rappelant régulièrement que sa pièce n'a pas à être jugée selon les lois du théâtre. Qu'est-ce donc qu'un spectacle visuel de personnages animés qui admet un spectateur seulement par effraction et qui ne se joue pas sur une scène (tréteaux ou estrade)?

\section{L'invention des Lumières}

Que le cinéma soit l'invention des Lumières, il semble qu'on l'ait toujours su (on se sera seulement trompé en l'écrivant au singulier). Mais que Diderot ait pu parler du cinéma, on le sait avec certitude depuis un texte célèbre d'Eisenstein précisément intitulé «Diderot a parlé de cinéma» (1943) ${ }^{13}$, signalé en son temps par Roland Barthes dans un article non moins fameux ${ }^{14}$, et commenté à nouveaux frais par Denis Guénoun ${ }^{15}$. Le réalisateur russe, lui-même passé du théâtre au cinéma, y a ce mot proprement abyssal: «Le cinéma est le fils naturel du théâtre» le cinéma est un bâtard et non pas un descendant légitime de l'art dramatique.

Si les historiens du pré-cinéma ne remontent généralement pas endeçà des spectacles révolutionnaires ${ }^{16}$, en offrant ainsi au septième art

I2. Cf. notamment les articles de D. Guedj, "Les drames de Diderot» et de J.-L. Haquette, «Le public et l'intime».

I3. Nous nous référons à la traduction établie en 1986 par F. Albéra et N. Kleinman. Une première traduction française avait été donnée deux ans plus tôt dans le numéro 661 de la revue Europe sous le titre: "Diderot a parlé de cinéma», mais amputée des développements centraux relatifs à la formation des acteurs de cinéma; c'est cette version tronquée que commente J.-C. Bonnet dans "Diderot a inventé le cinéma", p. 27-33.

I4. R. Barthes, «Diderot, Brecht, Eisenstein».

I5. D. Guénoun, Le Théâtre est-il nécessaire?, p. 129-141.

I6. H. El Nouty, Théatre et pré-cinéma, dont le chap. I porte sur la «cérémonie révolutionnaire». Cf. toutefois M. Chatel de Brancion, Le cinéma au siècle des Lumières. 
un siècle de pré-histoire, le "cinématisme» d'Eisenstein, qui invite à faire du cinéma "un point de vue privilégié pour penser ces arts connexes, déceler en eux des techniques ou des pratiques dont le potentiel n'est pleinement réalisé que par le cinéma ${ }^{17}$, autorise à lire Diderot depuis le cinéma, pour reconnaître dans Le Fils naturel «l'anticipation d'un théâtre qui ne peut se réaliser que grâce à son descendant naturel, le cinéma" ${ }^{18}$.

Telle est la démarche esquissée par Eisenstein tout au long d'un article rédigé avec une allégresse qui confine à la désinvolture, mais qui nous vaut de loin en loin un minutieux relevé (Eisenstein lit Diderot en français) de ces moments des Entretiens et de l'essai De la Poésie dramatique, où la quête de Diderot est un désir de cinéma.

Je n'en donnerai ici que quelques échantillons - autant d'occasions de relire avec le regard du cinéma (et non plus l'œil du théâtre) quelques formules bien connues, qui valent comme autant de "prévisions cinématographiques» ${ }^{19}$ de la part de Diderot.

\section{L'ail-caméra}

Il faut d'abord prendre acte de la situation qui est celle de l'unique spectateur, entré par effraction sur le lieu de ce qui n'est pas une représentation de théâtre. Ce "coin" d'où l'on peut tout entendre et tout voir sans être vu, ce lieu focal en fonction duquel le «salon" a été préalablement "rangé», et qui place le spectateur de plain-pied avec des protagonistes qui doivent ignorer continûment sa présence, qu'est-ce donc sinon l'objectif de la caméra? Soulignons au passage que ce désir d'un affaissement de la scène est exactement contemporain des débats relatifs à la possibilité d'asseoir les spectateurs du parterre ${ }^{20}$.

I7. J.-L. Jeannelle, «Homo cinematographicus». Dans le même article, le cinématisme se trouve défini comme "une idée du cinéma indépendante du dispositif technique qui en assure la diffusion et identifiable dans toute une série d'arts proches, tels que la peinture, l'architecture, la littérature ou la musique».

I8. S. Eisenstein, «Diderot a parlé de cinéma», p. 78.

19. Ibid.

20. Cf. M. Escola, «Descendre au parterre (Déloger le public de théâtre)». 


\section{Le mouvement de l'action}

Il faut remarquer ensuite que le premier des trois Entretiens, qui porte d'abord sur l'unité de lieu, ne dénonce pas la convention en tant que telle: Dorval ne professe pas tant une rupture à l'égard de l'unité de lieu qu'une émancipation à l'égard de la contrainte du décor unique:

Ah! si nous avions des théâtres où la décoration changeât toutes les fois que le lieu de la scène doit changer. [...] Le spectateur suivrait sans peine tout le mouvement d'une pièce; la représentation en deviendrait plus variée, plus intéressante et plus claire ${ }^{21}$.

Le vœu n'est pas ici de s'affranchir des limites matérielles de la scène: il est de substituer à la succession des scènes la possibilité de rendre le «mouvement" par "le montage de l'action d'un point à l'autre», écrit Eisenstein - par quoi le «salon» de Diderot constitue tout autre chose qu'«une reductio ad absurdum de la doctrine tristement célèbre» de l'unité de lieu.

Pour Diderot, l'idéal de l'«unité de lieu» n'était en rien synonyme de «magma» d'événements en un lieu de l'action - mais de lieu unique pour chacune de ses phases. C'est-à-dire ce qui se trouve déjà à la base du travail du metteur en scène au cinéma, lequel choisit à chaque nouveau cadre le seul point où l'événement se déroulant réellement va se fixer avec le plus de précision dans les consciences ${ }^{22}$.

\section{L'intuition du travelling}

Il faut ensuite prêter, sinon reconnaître à Diderot l'intuition du travelling, parfaitement décelable dans les précisions délivrées par Dorval quant aux lieux "réels" où se sont déroulés les dialogues (eux-mêmes supposés authentiques), dont les scènes IV, 3 et V, 5 sont la transposition dramatique:

2I. D. Diderot, Le Fils naturel, p. 72 sq.; cité par S. Eisenstein, «Diderot a parlé de cinéma", p. 92.

22. S. Eisenstein, «Diderot a parlé de cinéma», p. 93. 
Je me promenais avec Constance dans cette grande allée, sous les vieux marronniers que vous voyez, lorsque je demeurai convaincu qu'elle était la seule femme qu'il y eût au monde pour moi. [...] [IV, 3]

Au premier bruit de l'arrivée de mon père, nous descendîmes, nous accourûmes tous; et la dernière scène $[\mathrm{V}, 5]$ se passa en autant d'endroits différents que cet honnête vieillard fit de pauses, depuis la ported'entrée jusque dans ce salon. Je les vois encore, ces endroits... Si j'ai renfermé toute l'action dans un lieu, c'est que je le pouvais sans gêner la conduite de la pièce, et sans ôter de la vraisemblance aux événements ${ }^{23}$.

Eisenstein, qui cite le passage in extenso, en donne ce commentaire:

Diderot n'a même pas eu [i.e. n'a pas eu seulement] la vision d'un déplacement par le montage de l'action d'un point à l'autre, mais celle de toute une salle de spectacle qui suive pas à pas de manière invisible les péripéties du dialogue (c'est-à-dire faire ce qu'accomplit sans peine le ciné-panorama ${ }^{24}$ glissant sur les rails, soit dit en passant une forme camouflée de montage, le plus souvent appréciée de ceux qui reculent devant le montage à découvert dans la construction d'une scène ou d'un dialogue) ${ }^{25}$.

\section{Théorie du cadre}

Ce qui se cherche encore sous le nom de "tableau", dans les Entretiens mais un peu partout ailleurs dans l'œuvre de Diderot, c'est une théorie du cadre, notion capitale dans la pensée comme dans l'enseignement d'Eisenstein :

Un incident imprévu qui se passe en action, et qui change subitement l'état des personnages, est un coup de théâtre.

Une disposition des personnages sur la scène, si naturelle et si vraie, que rendue fidèlement par un peintre, elle plairait sur la toile, est un tableau $^{26}$.

23. D. Diderot, Le Fils naturel, p. 73 sq.

24. "Kino-panorama: ce terme désigne, dans les années vingt, tout mouvement d'appareil (panos ou travellings - ce dernier mot n'existant pas en russe). Actuellement le terme en usage est: déplacement" (Note des éditeurs).

25. S. Eisenstein, «Diderot a parlé de cinéma », p. 92. La citation de Diderot est donnée à la suite, p. 93.

26. D. Diderot, Premier entretien sur Le Fils naturel, p. 79. M. Fried (La place du spectateur, p. 99) commente la formule en ces termes: "un coup de théâtre se produit 
Le philosophe interlocuteur de Dorval reconnaît aussitôt un tableau dans le souvenir qu'il garde de la scène II, 4 («le malheureux Clairville, renversé sur le sein de son ami, comme dans le seul asile qui lui reste...»), s'attirant cette remarque de Dorval:

Convenez que ce tableau n'aurait point eu lieu sur la scène; que les deux amis n'auraient osé se regarder en face, tourner le dos au spectateur, se grouper, se séparer, se rejoindre; et que toute leur action aurait été bien compassée, bien empesée, bien maniérée, et bien froide.

La suite immédiate du passage dans le premier Entretien invite à penser l'action dramatique comme une succession de tableaux:

Si un ouvrage dramatique était bien fait et bien représenté, la scène offrirait au spectateur autant de tableaux réels qu'il y aurait dans l'action de moments favorables au peintre ${ }^{27}$.

«Il parle d'or!», fait valoir Eisenstein, qui cite l'intégralité du passage avant de détailler sa propre manière de travailler, laquelle consiste à "découper par le rectangle du cadre un fragment d'événement vivant» ${ }^{28}$.

du dedans de l'action; il provoque un brusque changement dans la conscience des personnages en jeu. La disposition des personnages et les propriétés scéniques qui constituent le tableau sont au contraire extérieurs à l'action, au point que les personnages eux-mêmes semblent inconscients de l'existence de cette dernière et qu'ils ignorent son effet sur le public. La fonction du tableau pour Diderot serait ainsi de neutraliser les capacités de visualisation du public».

27. D. Diderot, Premier entretien sur Le Fils naturel, p. 80.

28. S. Eisenstein, «Diderot a parlé de cinéma», p. 95 pour la citation. Diderot fournit ainsi à Eisenstein l'occasion de distinguer deux «techniques de composition du cadre» (ibid., p. 93): "Soit la “découpe" par le rectangle du cadre d'un fragment de l'événement vivant. Soit "le remplissage" du rectangle du cadre par les éléments du milieu, d'actions et d'objets». La deuxième technique est propre aux "tréteaux" ("cadre de scène»). La première, assimilée au "salon» est préférable: "En remplissant dans les croquis de réalisateur le rectangle du cadre, j'envisage toujours, pour ma part, environnement, décor et personnages comme des événements réels d'où j’arrache avec le rectangle du cadre ce qui est essentiel à un moment décisif de l'action. De manière générale, je préfère et pratique surtout la recherche de points décisifs pour la caméra - résolvant l'événement dramatique par le principe des quatre murs, sans faire dépendre la conception de celui-ci d'un point préalablement établi» (ibid., p. 94). 


\section{Le principe du montage}

Au fond, ce qui se cherche dans les Entretiens, c'est la seule chose dont le cinéma pourra légitimement revendiquer l'invention: le principe du montage. Eisenstein épingle dans l'essai De la poésie dramatique, qu'il lit aussi bien que les Entretiens dans le texte français, cette phrase bien faite pour le réjouir:

[...] le spectateur [devrait être] au théâtre comme devant une toile où des tableaux divers se succèderaient par enchantement $[\ldots]^{29}$.

Eisenstein met encore au crédit d'une intuition par Diderot de l'art du montage cette réforme du lieu théâtral que préconise Dorval dans le second Entretien:

[Sur nos théâtres], on ne peut jamais montrer qu'une action, tandis que dans la nature il y en a toujours de simultanées, dont les représentations concomitantes, se fortifiant réciproquement, produiraient sur nous des effets terribles. [...] Ils attendent, pour se montrer, un homme de génie qui sache combiner la pantomime avec le discours, entremêler une scène parlée avec une scène muette, et tirer parti de la réunion de deux scènes, et surtout de l'approche ou terrible ou comique de cette réunion qui se ferait toujours (i.e. sans discontinuité ou rupture de la liaison des scènes) ${ }^{30}$.

Nul doute qu'Eisenstein ne se soit spontanément rangé parmi ces hommes de génie. Il suffit de se plonger dans ses écrits, et tout particulièrement dans ses leçons sur Balzac, Zola et Dostoïevski, pour comprendre qu'il partageait avec le Diderot des Salons une imagination visuelle sérielle, qu'il faut dire littéralement cinémato-graphique plutôt que picturale:

[à force de regarder les productions des peintres], j’ai pris l'habitude d'arranger mes figures dans ma tête comme si elles étaient sur la toile;

29. D. Diderot, De la poésie dramatique, p. 257.

30. D. Diderot, Second entretien sur Le Fils naturel, p. 101. Suit un «exemple domestique et commun", où l'on suppose qu'un spectateur peut assister successivement à la réaction du père et de la mère à l'annonce de la mort de leur fils, avant leur réunion dans une scène commune (Ibid., p. 102 sq.). 
$[\ldots]$ je les y transporte, et [...] c'est sur un grand mur que je regarde quand j'écris (Salon de 1767) ${ }^{31}$.

\section{Synchronisation}

Eisenstein aurait encore pu trouver chez Diderot l'idée de la synchronisation, comme l'a suggéré J.-C. Bonnet ${ }^{32}$ en citant le célèbre passage de la Lettre sur les sourds et muets (1731), où le philosophe propose d'éprouver la valeur d'un spectacle en se bouchant les oreilles puis en fermant les yeux alternativement

[...] si pour juger sainement de l'intonation, il faut écouter le discours sans voir l'acteur, il est tout naturel de croire que pour juger sainement du geste et des mouvements, il faut considérer l'acteur sans entendre le discours ${ }^{33}$.

On pourrait verser encore au bénéfice d'une pensée de la synchronisation le célèbre développement du second Entretien ${ }^{34}$ sur les bienfaits de la pantomime ("Nous parlons trop dans nos drames; et conséquemment, nos acteurs n'y jouent pas assez»), et y lire comme une préfiguration des exigences du cinéma muet.

\section{Sortir du jeu: conditions d'une déposition du jeu}

Mais c'est sur la question du jeu de l'acteur qu'Eisenstein trouve en Diderot un peu mieux qu'un précurseur; l'auteur des Entretiens sur Le Fils naturel lui apparaît comme un recours au moment où il doit fonder une méthode de formation au métier d'acteur de cinéma à l'Institut national de la cinématographie S. A. Guerassimov (VGIK), déplacé à Alma-Ata pendant l'invasion allemande. À la notable exception de Denis Guénoun, la chose a été mal perçue par les commentateurs français récents de l'article d'Eisenstein (qui y accèdent presque tous, il

3I. J.-C. Bonnet, «Diderot a inventé le cinéma», p. 29.

32. Ibid., p. 29 sq.

33. D. Diderot, Lettre sur les sourds et muets, p. 100 sq.

34. S. Eisenstein, "Diderot a parlé de cinéma», p. 89 sq. 
est vrai, dans la version tronquée de 1984) ${ }^{35}$; l'essentiel des réflexions d'Eisenstein vise en effet à résoudre un problème et un seul: comment empêcher les futurs acteurs de cinéma de jouer comme des comédiens de théâtre? Comment les amener à sortir du jeu?

\section{À l'écran, les acteurs jouent mal.}

Même lorsqu'ils jouent bien.

Sauf exception très rares [...], le jeu cinématographique continue d'être le «fils naturel» du jeu théâtral.

Non au vrai sens de "naturel», hélas, mais au sens d'«illégitime».

Plus exactement - d'illicite au cinéma.

Sauf rares exceptions - il s'agit encore de théâtre photographié. [...]

[Ce jeu] n'offre pas encore l'authenticité de la vie, l'authenticité de l'émotion, le morceau de réalité - fût-il obtenu dans d'artificielles conditions de laboratoire - que peut et qu'a pour vocation de recréer l'art du cinéma.

Le fait de la recréation artificielle en soi ne nie nullement la possibilité de l'authenticité des résultats.

La contrefaçon est répugnante. La recréation est superbe. Le beurre contrefait la margarine - est aussi repoussant que le plagiat.

La glace artificiellement reconstituée est pleine du charme froid de la glace réelle ${ }^{36}$.

Ce qu'Eisenstein cherche et trouve chez Diderot, ce sont finalement les conditions qui obligeront les apprentis comédiens à se déprendre du jeu. On pourrait parler d'une «déposition» du jeu, comme on se défait d'une fonction, préalable à l'" exposition " des acteurs au dispositif de captation de la caméra et à leur imposition ultérieure sur la surface de l'écran. Le théoricien russe parle d'un travail de «décapage» à opérer pour chacun d'eux.

Eisenstein nous invite ainsi à lire dans la fiction théorique du Fils naturel une pensée du non-jeu; et c'est encore un paradoxe: alors même que Diderot est l'exact contemporain des deux premiers traités sur l'art du comédien - Le Comédien de Rémond de Saint-Albine (1747) et L’Art $d u$ théâtre d'Antoine-François Riccoboni $(1750)^{37}$-, il n'a pas même

35. Cf. supra, n. 12.

36. S. Eisenstein, "Diderot a parlé de cinéma», p. 79.

37. Tous deux commentés par D. Guénoun en regard du Paradoxe dans Le Théâtre est-il nécessaire?, p. 60 sq. 
cherché à les lire durant l'entreprise de 1757 , et il ne l'a fait qu'après coup $^{38}$. C'est bien que dans la fable du Fils naturel, Diderot ne cherche pas à réformer le jeu, mais plutôt à théoriser un non-jeu, soit à penser un mode d'actualisation (du passé familial, de la fiction dramatique) qui n'est pas celui de la re-présentation théâtrale, qui n'est pas de l'ordre de l'incarnation d'un personnage, qui n'opère donc pas sur le mode du «comme si ». Diderot a ainsi un peu plus qu'esquissé la différence entre le jeu du comédien de théâtre et celui de l'acteur de cinéma.

J'examinerai pour finir ces conditions de déposition du jeu qu'Eisenstein identifie dans les textes de Diderot.

\section{Un jeu sans adresse: un spectacle sans spectateur}

La première de ces conditions est bien connue: le théâtre de Diderot est un spectacle sans spectateur, et le jeu de ses comédiens est un jeu sans adresse, dès lors qu'il ne requiert pas la présence d'un public assemblé. Les formules sont célèbres et partout citées; la première intervient lorsqu'il s'agit de proscrire l'usage des tirades:

Dans une représentation dramatique, il ne s'agit non plus du spectateur que s'il n'existait pas. Y a-t-il quelque chose qui s'adresse à lui? Lauteur est sorti de son sujet, l'acteur entraîné hors de son rôle. Ils descendent tous deux du théâtre. Je les vois dans le parterre; et tant que dure la tirade, l'action est suspendue pour moi, la scène reste vide ${ }^{39}$.

La seconde figure dans l'essai De la Poésie dramatique, et elle est plus célèbre encore:

Et l'acteur, que deviendra-t-il, si [comme auteur] vous vous êtes occupé du spectateur? Croyez-vous qu'il ne sentira pas que ce que vous avez placé dans cet endroit et dans celui-ci n’a pas été imaginé pour lui? Vous avez pensé au spectateur, il s'y adressera. Vous avez voulu qu'on vous applaudît, il voudra qu'on l'applaudisse; et je ne sais plus ce que l'illusion deviendra.

38. Il les mentionne tous deux, mais seulement dix ans plus tard, dans Le Paradoxe, p. 328.

39. D. Diderot, Second entretien sur Le Fils naturel, p. 91. 
J'ai remarqué que l'acteur jouait mal tout ce que le poète avait composé pour le spectateur; et que, si le parterre eût fait son rôle, il eût dit au personnage: "À qui en voulez-vous? Je n'en suis pas. Est-ce que je me mêle de vos affaires? Rentrez chez vous"; et que si l'auteur eût fait le sien, il serait sorti de la coulisse, et eût répondu au parterre: "Pardon, messieurs, c'est ma faute; une autre fois je ferai mieux, et lui aussi». Soit donc que vous composiez, soit que vous jouiez, ne pensez non plus au spectateur que s'il n'existait pas. Imaginez sur le bord du théâtre un grand mur qui nous sépare du parterre; jouez comme si la toile ne se levait pas ${ }^{40}$.

Cette dernière phrase est citée en russe par Eisenstein, et placée en épigraphe de son article. Il rappelle que les recherches du «jeune Théâtre d'Art» de Stanislavski furent étroitement liées à ce postulat d'un "quatrième mur»:

[le Théâtre d'Art] nourrissait le rêve que l'on vive et se comporte sur scène comme dans une chambre. On y aurait parlé comme on parle, marché comme on marche, senti comme on sent. [...] On y aurait agi de manière que l'un des quatre murs de clôture soudain béant puisse permettre l'intrusion d'un regard dans le petit monde de ce qui se passait sur scène ${ }^{41}$.

Conviction toute «illusoire» selon Eisenstein, et sans effets sur le jeu des comédiens: le théâtre se révèle structurellement inapte à abolir le fait même de l'adresse, à "pratiquer cet abandon de la direction imposée par la présence du public», selon le mot de D. Guénoun ${ }^{42}$. Le Théâtre d'art a échoué à donner corps sur scène au rêve de Diderot, que seul le cinéma pouvait réaliser.

Le cinématographe, c'est justement le seul domaine où peuvent se réaliser les rêves de ceux qui déliraient à propos d'un "quatrième mur " irréalisable au théâtre ${ }^{43}$.

40. D. Diderot, De la Poésie dramatique, $\$ 11$, p. 210 sq.

4I. S. Eisenstein, "Diderot a parlé de cinéma», p. 83. Eisenstein poursuit par une allusion au Diable boiteux de Cazotte (1707), où Asmodée soulève les toits des maisons pour révéler la vie et les secrets des hommes au jeune homme qu'il se propose d'instruire.

42. D. Guénoun, Le théâtre, est-il nécessaire?, p. 134.

43. S. Eisenstein, "Diderot a parlé de cinéma», p. 87. 
Ce qui est irréalisable au théâtre, ce n'est pas de poser la transparence d'un quatrième mur: c'est d'abolir la présence même du public. Qu'est-ce qui distingue en effet pour un acteur un plateau de cinéma d'une scène théâtrale? C'est de ne pas savoir d'où il est regardé, que le regard sur lui ne vienne pas toujours du même point, et ne se tienne pas à une distance constante. Eisenstein rapporte en note l'un des "rêves" ultimes de Stanislavski :

Dans les dernières années, K. S. Stanislavski disait ne pas aimer les mises en scène préparées à l'avance. Il rêvait d'une scène où les quatre murs auraient été mobiles et où l'acteur n'aurait pas su lequel s'escamoterait durant le spectacle ${ }^{44}$.

\section{L'abandon de la frontalité}

Il se trouve que cette scène débarrassée de sa frontalité, et qui commence à ressembler à un plateau de cinéma, Diderot en a tracé les plans le premier encore, dans la lettre déjà citée, adressée en 1758 à la comédienne (et romancière) Marie-Jeanne Riccoboni et qu'Eisenstein ne pouvait pas connaître:

Je voudrais que vous eussiez pour vos répétitions un théâtre particulier, tel par exemple qu'un grand espace rond ou carré, sans devant, ni côtés, ni fond, autour duquel vos juges seraient placés en amphithéâtre. Je ne connais que ce moyen de vous dérouter ${ }^{45}$.

Pour "dérouter» définitivement le comédien, pour priver son jeu de toute direction et de la possibilité même de son adresse, il ne suffit pas d'effondrer l'un des murs, comme dans Le Charme discret de la bourgeoisie de L. Buñuel, ni même de les escamoter tour à tour: il faut encore "effondrer les tréteaux" selon le mot d'Eisenstein pour parvenir à la complète exposition de l'acteur. Le réalisateur se réjouit ainsi qu'à l'Institut de cinéma d'Alma Ata, on ait "démoli les tréteaux» (depuis janvier 1943):

On y a démoli les tréteaux: l'action scénique a été portée en milieu muré de quatre côtés.

44. Ibid., p. 83.

45. D. Diderot, Entretiens sur Le Fils naturel, p. 348. 
Le public est assis de tous les côtés.

Le metteur en scène circule: repérant le point de démonstrativité maximale du côté précis où l'action prend le plus de relief - une action qui ne cherche pas à être démonstrative par rapport à tel ou tel côté ${ }^{46}$.

Les apprentis acteurs de cinéma doivent abolir en eux jusqu'à la perception de la scène, "détrui[re] l'estrade, anéanti[r] les tréteaux dans les limites de leur propre technique de jeu ${ }^{47}$; Eisenstein détaille ainsi longuement l'impact de l'abandon de la frontalité ${ }^{48}$.

46. Ibid., p. 89. Il rappelle que dès 1920, l'espace scénique du studio du Théâtre d'Art "n'était pas même surélevé par rapport aux sièges des spectateurs" (Ibid., p. 83).

47. Ibid., p. 88.

48. "Ceux qui "brillaient" sur scène, qui semblaient vivants et convaincants sur les tréteaux - se sont soudain révélés fort loin de la vie. D’autre part, ceux qui avaient du mal à s'ouvrir, à se déployer, s'aplatir derrière la rampe - débordaient d'une vie intérieure splendide. Le grand renfort de jeux, de tours de force scéniques a fait place à une fine recherche de détails que jamais personne ne remarquerait sur scène, mais que la caméra sera heureuse de fixer en gros plan. Et ces détails ne surgissent pas parce que quelqu'un pense à la caméra. Il n'en est rien: ce sont précisément ces détails dans lesquels naturellement s'enveloppent les états affectifs naturels» (S. Eisenstein, "Diderot a parlé de cinéma", p. 89). On rapprochera le constat de celui formulé par Diderot dans le Paradoxe (p. 326 sq.) sur une actrice débutante: "C'est une expérience qu'apparemment vous aurez faite quelquefois, lorsque appelé par un débutant ou par une débutante, chez elle, en petit comité, pour prononcer sur son talent, vous lui aurez accordé de l'âme, de la sensibilité, des entrailles, vous l'aurez accablée d'éloges et l'aurez laissée, en vous séparant d'elle, avec l'espoir du plus grand succès. Cependant qu'arrive-t-il? Elle paraît [sur le théâtre], elle est sifflée, et vous vous avouez à vous-même que les sifflets ont raison. D'où cela vient-il ? Est-ce qu'elle a perdu son âme, sa sensibilité, ses entrailles, du matin au soir? Non; mais à son rez-de-chaussée vous étiez terre à terre avec elle; vous l'écoutiez sans égard aux conventions, elle était vis-à-vis de vous, il n'y avait entre l'un et l'autre aucun modèle de comparaison; vous étiez satisfait de sa voix, de son geste, de son expression, de son maintien; tout était en proportion avec l'auditoire et l'espace; rien ne demandait de l'exagération. Sur les planches tout a changé: ici il fallait un autre personnage, puisque tout s'était agrandi. Sur un théâtre particulier, dans un salon où le spectateur est presque de niveau avec l'acteur, le vrai personnage dramatique vous aurait paru énorme, gigantesque, et au sortir de la représentation vous auriez dit à votre ami confidemment: "Elle ne réussira pas, elle est outrée"; et son succès au théâtre vous aurait étonné. Encore une fois, que ce soit un bien ou un mal, le comédien ne dit rien, ne fait rien dans la société précisément comme sur la scène; c'est un autre monde». Le passage se trouve commenté par D. Guénoun, Le théâtre, est-il nécessaire?, p. 80 $s q$. 
La réduction de la scène au "salon" dans les Entretiens sur Le Fils naturel («Laissez là les tréteaux, rentrez dans le salon») ${ }^{49}$, qui constitue un milieu muré de quatre côtés, n'a pas d'autre fonction que de permettre une telle exposition du comédien à un regard toujours imprévu.

\section{Au milieu du jeu}

Poser, dans la fiction de la cérémonie familiale, que les comédiens ne jouent que pour eux, c'est signifier que le jeu se joue, littéralement, entre eux: le jeu de chacun n'est adressé qu'à son partenaire, le jeu n'est plus porté au-devant mais il reste entre eux, où il doit être capté par un dispositif installé non pas face à eux mais au milieu d'eux. On trouve chez Diderot comme chez Eisenstein une même insistance à situer le jeu au milieu des personnages: le jeu se tient entre les personnages, au lieu de se déployer dans l'intervalle qui sépare la scène des gradins.

Eisenstein envisage la différence entre l'écriture dramatique et l'écriture cinématographique comme "passage de l'événement noté unilatéralement à l'événement encerclé» — par quoi une scène de roman réaliste se prête mieux au cinéma qu'une scène de théâtre. Diderot invite quant à lui le dramaturge à «se renfermer entre les personnages et [à] laisser le spectateur devenir ce qu'il voudra " ${ }^{50}$, à faire donc en sorte que "tout l'intérêt [soit] relatif aux personnages ${ }^{51}$. On méditera pour finir à cette

49. D. Diderot, Premier entretien sur Le Fils naturel, p. 76 (cf. aussi, ibid., p. 77 : «Ce n'est pas [au théâtre], c'est au salon qu'il faut juger mon ouvrage»); et Second Entretien, p. 98: "Je gage que vous me voyez encore sur la scène française, au théâtre - Vous croyez que votre ouvrage ne réussirait point au théâtre? - Difficilement. Il faudrait ou élaguer en quelques endroits le dialogue, ou changer l'action théâtrale et la scène. - Qu'appelez-vous changer la scène? - En ôter tout ce qui resserre un lieu déjà trop étroit; avoir des décorations, pouvoir exécuter d'autres tableaux que ceux qu'on voit depuis cent ans; en un mot, transporter au théâtre le salon de Clairville, comme il est». Cf. également p. 110 où Dorval répond en ces termes au philosophe-spectateur qui incriminait la longueur de la scène IV, 3 entre lui-même et Constance: «Ah! vous voilà remonté sur la scène. [...] Vous nous voyez, Constance et moi, sur le bord d'une planche, bien droits, nous regardant de profil, et récitant alternativement la demande et la réponse. Mais est-ce ainsi que cela se passait dans le salon? Nous étions tantôt assis, tantôt droits; nous marchions quelquefois. Souvent nous étions arrêtés, et nullement pressés de la fin d'un entretien qui nous intéressait tous deux également».

50. D. Diderot, De la Poésie dramatique, p. 210 sq.

5I. Ibid., p. 209: «Si l'on avait conçu que, quoiqu'un ouvrage dramatique ait été fait pour être représenté, il fallait cependant que l'auteur et l'acteur oubliassent le specta- 
réflexion d'Eisenstein, qui forme la suite de la précédente citation et où se résume l'essentiel de sa méthode de formation au métier d'acteur de cinéma :

Le metteur en scène circule [parmi les apprentis comédiens, sur le plateau muré des quatre côtés] [...]. La mise en scène, le jeu, l'interaction et la mise en place émergent entièrement de l'intérieur des relations entre les personnages [,] de l'intérieur de leur communication avec le milieu, l'entourage.

Lacteur ignore d'où peut le fixer l'œil de l'objectif: il ne pense pas à lui mais à son partenaire avec lequel il est lié par le jeu; il ne pense pas à la rampe ou aux coulisses, mais à la porte qui l'attire; à la fenêtre par laquelle il a envie de regarder; au divan sur lequel il a envie de s'étendre ${ }^{52}$.

\section{Abandonner le texte}

Selon le principe déjà rappelé qui veut que «tout l'intérêt [doit être] relatif aux personnages", sans nul souci des spectateurs, et en vertu de la fiction qui veut que chaque personnage du drame joue son propre rôle, Diderot en vient à envisager le pré-texte dramatique comme un texte sans autorité: un scénario amendable selon les suggestions de chaque comédien, qui peut "ajout[er]» ou "retranch[er]" à son rôle; "Il y a des endroits qu'il faudrait presque abandonner à l'acteur. C'est à lui à

teur, et que tout l'intérêt fût relatif aux personnages, on ne lirait pas si souvent dans les poétiques: Si vous faites ceci ou cela, vous affecterez ainsi ou autrement votre spectateur. On y lirait au contraire: Si vous faites ceci ou cela, voici ce qui en résultera parmi vos personnages. Ceux qui ont écrit de l'art dramatique ressemblent à un homme qui, s'occupant des moyens de remplir de trouble toute une famille, au lieu de peser ces moyens par rapport au trouble de la famille, les pèserait relativement à ce qu'en diront les voisins. Eh! laissez là les voisins; tourmentez vos personnages; et soyez sûr que ceuxci n'éprouveront aucune peine que les autres ne partagent".

52. S. Eisenstein, "Diderot a parlé de cinéma», p. 89. Le principe est reformulé plusieurs fois: "L'itinéraire superstructurel des points de prise de vue imposant alors ses arabesques au développement d'une action (entre quatre murs!) dont les volte-face, les péripéties, les positions et déplacements se définissent et s'engendrent de l'intérieur de ce qui se passe et procèdent des déterminations internes de l'action» (Ibid., p. 94); il parle encore d'une "juste compréhension de la composition du jeu se développant à partir de la croissance d'un point intérieur et non de ce point d'avance cloué à la surface préétablie de l'écran" (ibid.). 
disposer de la scène écrite, à répéter certains mots, à revenir sur certaines idées, à en retrancher quelques-unes, et à en ajouter d'autres» ${ }^{53}$.

\section{Le legs $d u$ Fils naturel}

La réduction de la "scène" au "salon", qui constitue le leitmotiv des Entretiens sur Le Fils naturel, a donc pour principale fonction de théoriser les conditions d'une "déposition" du jeu théâtral au bénéfice de ce que j’ai appelé imprudemment un «non-jeu», soit l'exposition à une dynamique interne à l'action représentée, qui fait émerger le jeu à l'intérieur des relations entre les personnages où il ne peut être que surpris par le regardant. Ce jeu sans adresse ni direction, c'est celui dont le cinéma a besoin. Tel est le legs de Diderot identifié par Eisenstein. Le jeu est ainsi le seul domaine où le cinéma doit impérativement s'affranchir de sa filiation avec l'art dramatique, pour se revendiquer comme «fils naturel du théâtre».

Marc Escola

Université de Lausanne

53. D. Diderot, Premier entretien sur Le Fils naturel, p. 77 et Second entretien sur Le Fils naturel, p. 90. 


\section{BIBLIOGRAPHIE}

\section{Textes}

Diderot, Denis, Le Fils naturel, Le Père de famille, Est-il bon? Est-il méchant?, présentation, notes, annexes, chronologie et bibliographie par Jean Goldzink, Paris, GF-Flammarion, 2005.

—, Entretiens sur Le Fils naturel, De la Poésie dramatique, Paradoxe sur le comédien, présentation, notes, annexes, chronologie et bibliographie par Jean Goldzink, Paris, GF-Flammarion, 2005.

—, Lettre sur les aveugles à l'usage de ceux qui voient. Lettre sur les sourds et muets à l'usage de ceux qui entendent et qui parlent, présentation, notes, dossier, chronologie, bibliographie par Marian Hobson, Simon Harvey, Paris, GF-Flamarion, 2000.

\section{Travaux}

BARThes, Roland, "Diderot, Brecht, Eisenstein », in Id., L'obvie et l'obtus. Essais critiques III, Seuil, 1982, p. 86-93 (première publication in Cinéma, Théoriellecture, Revue d'Esthétique, no. spécial, 1973).

Bonnet, Jean-Claude "Diderot a inventé le cinéma", Recherches sur Diderot et sur l'Encyclopédie, 18-19 (1995), p. 27-33.

Chatel de Brancion, Laurence, Le cinéma au siècle des Lumières, SaintRémy-en-L’Eau, M. Hayot, 2007.

Eisenstein, Sergej, "Diderot a parlé de cinéma", in Le mouvement de l'art, texte établi par François Albéra et Naoun Kleinman, Paris, Éd. du Cerf, 1986 (1943), p. 77-96.

El Nouty, Hassan, Théâtre et pré-cinéma. Essai sur la problématique du spectacle au XIX $X^{e}$ siècle, Paris, A.-G. Nizet, 1978.

Escola, Marc, "Dans le cristal du temps. Diderot, Deleuze, le théâtre, le cinéma», in Stéphane Lojkine, Adrien Paschoud, Barbara 
Selmeci-Castioni (éds), Diderot et le temps, Aix-en-Provence, Presses universitaires de Provence, 2016, p. 203-212.

—, "Ce théâtre n'est pas le nôtre”. Rousseau et Diderot spectateurs de plein air", Revue d'Historiographie du théatre, 3 (2017) <https:// sht.asso.fr/8294-2/>.

—, "Descendre au parterre (Déloger le public de théâtre)", communication présentée au colloque Hypothèses sur le théâtre, la politique, l'Europe, la philosophie. Avec Denis Guénoun, Lausannel Genève, 2-4 novembre 2017, sous la direction d'Éric Eigenmann, Marc Escola, Martin Rueff, à paraître aux éditions MétisPress, à Genève.

Fried, Michael, La place du spectateur, Paris Gallimard, 1990 (1980).

GuedJ, Denis, «Les drames de Diderot», Diderot Studies, 14 (1971), p. 15-95.

GuÉnoun, Denis, Le théâtre, est-il nécessaire?, Circé, 1997.

Haquette, Jean-Louis, "Le public et l'intime. Réflexions sur le statut du visible dans Le Fils naturel et les Entretiens", in Marc Buffat (dir.), Diderot, l'invention du drame, Paris, Klincksieck, 2000, p. 59-76.

Jeannelle, Jean-Louis, "Homo cinematographicus: sur l'hypothèse d'une préhistoire du cinéma", présenté dans le cadre du séminaire DSA-LILA (École normale supérieure, Paris): Anachronies: textes anciens et théories modernes (4-5 mai 2012), Actes publiés dans l'Atelier littéraire de Fabula <https://www.fabula.org/atelier. php?Homo_cinematographicus $>$. 
